\title{
Cultura y comunicación intercultural. Aproximaciones conceptuales
}

\author{
Marta Rizo' \\ Universidad Autónoma de la Ciudad de México \\ Vivian Romeu \\ Universidad Autónoma de la Ciudad de México
}

\begin{abstract}
RESUMEN: A partir de la revisión de varias acepciones de la cultura, este texto pretende relacionar este concepto con la comunicación, y especialmente con las situaciones de interculturalidad. La idea de cultura que presentamos intenta dar cuenta de los mecanismos de estructuración del mundo, de los modelos que sirven para dar sentido a la realidad. De ahí que en el texto adquieran gran relevancia las dimensiones cognitiva y comunicativa de la cultura. La interculturalidad, entendida necesariamente como un proceso comunicativo, es comprendida como la puesta en escena de culturas en contacto. El contacto, vaya encaminado a la negociación o al conflicto, se da siempre mediado por lo que llamamos fronteras internas.
\end{abstract}

\footnotetext{
${ }^{1}$ Doctora en Comunicación por la Universidad Autónoma de Barcelona (Espana). Actualmente, profesora-investigadora de la Academia de Comunicación y Cultura de la Universidad Autónoma de la Ciudad de México. Coordinadora de la Línea de Investigación en Comunicación Intercultural en esta institución. Miembro del Grupo hacia una Comunicología Posible (GUCOM, México) y de la Asociación Mexicana de Investigadores de la Comunicación (AMIC, México). Sus líneas de investigación son: Comunicología y teoría de la comunicación, Psicología y Comunicación, Comunicación intercultural, Comunicación y Ciudad. ${ }^{2}$ Maestra en Humanidades por el Tecnológico de Monterrey (México), Licenciada en Filosofía por la Universidad de la Habana (Cuba). Actualmente, profesora-investigadora de la Academia de Comunicación y Cultura de la Universidad Autónoma de la Ciudad de México. Coordinadora de la Línea de Investigación en Comunicación Intercultural en esta institución. Miembro de la Asociación Mexicana de Investigadores de la Comunicación (AMIC, México). Sus líneas de investigación son: Arte y Comunicación, Estética de la Recepción, Comunicación intercultural, Semiótica.
} 


\begin{abstract}
From the revision of several meanings of the culture, this text tries to relate this concept to the communication, and especially with the intercultural situations. The culture idea that we presented/displayed tries to give account of the mechanisms of structuring of the world, of the models that serve to give sense to the reality. For that reason in the text, they acquire great relevance the dimensions cognitive and communicative of the culture. The intercultural, understood necessarily like a communicative process, it is included/understood like the putting in scene of cultures in contact. The contact, go directed to the negotiation or the conflict, one occurs always halffull reason why we called internal borders..
\end{abstract}

\title{
Introducción
}

Los estudios sobre la cultura han sido abordados desde casi todas las disciplinas de las ciencias sociales; cada una de ellas ha ido marcando a lo largo del tiempo articulaciones específicas con aquélla, ofreciendo así un panorama multidisciplinar que intenta explicar las acciones de los individuos y los grupos humanos.

La comunicación siendo una disciplina que se ocupa de las significaciones a nivel enunciativo y receptivo de los mensajes ha sido, quizá, la gran aliada de los estudios sobre la cultura, toda vez que ésta es entendida como un sistema organizado donde las interacciones sociales tienen lugar, a partir de una serie de normativas y saberes que se articulan con el ámbito de los simbólico, y en específico con el ámbito del poder de lo simbólico.

Los vínculos entre la comunicación y los estudios sobre la cultura se deben en América Latina al legado de los conocidos y controvertidos Estudios Culturales; legado que puede concentrarse en tres aspectos fundamentales: el estudio de las llamadas "identidades colectivas", la inserción de objetos de estudio "plebeyos" como diría Roberto Follari- en la investigación sobre los fenómenos culturales, y uno muy importante, pero decididamente sesgado: el énfasis en los procesos y factores 
que condicionan la recepción en detrimento de los procesos y condiciones de enunciación de los mensajes.

Desde esta perspectiva, cultura y comunicación quedan entrelazadas, prácticamente de forma indisoluble, incluso indefinida. Ambas disciplinas hallan su convergencia a partir del núcleo simbólico que las caracteriza, de su estatuto significante, y de la operatividad de dicho estatuto en los espacios de la acción social.

Como bien dijera Paul Watzlawick (1971) es imposible no comunicar; cada gesto, cada acción, cada práctica sociocultural es significada por otros, es incluso entendida como intencional por otros, es decir, está siendo interpretada por los sujetos como un mensaje enunciado por alguien y para alguien, y esto obedece a la propia dinámica interaccional de la comunicación, a la dinámica de intercambio de información que necesaria y constitutivamente se da en toda situación comunicativa.

Como puede observarse, este axioma metacomunicativo estrecha los ya existentes vínculos entre la cultura y la comunicación, intentando esclarecer desde ahí la interrelación entre la interacción como base de la organización social y la interacción como sustento de la comunicación.

Partiendo de estos presupuestos conceptuales, la comunicación intercultural, como rama de estudio de la comunicación, expresa más que una relación de nombre con la cultura. Se trata de una comunicación donde se intercambian datos que son significados, evaluados e interpretados desde dos o más “ámbitos” culturales diferentes puesto que se da justamente en la interacción.

La cultura, desde estas coordenadas, precisa entonces de ser definida conceptualmente desde una perspectiva relacional y dialéctica; y eso es justamente lo que intentaremos demostrar en el acápite siguiente.

\section{Definiciones elementales de la cultura}

La cultura ha sido definida de innumerables formas. Es un concepto que comparte con otros - como la identidad, la sociedad y la comunicación, por citar algunos- el haber sido abordado desde múltiples perspectivas. Pese a la riqueza que esta multiplicidad encierra en sí misma, puede señalarse también un riesgo: la ambigüedad conceptual. 
Para la comunicación intercultural, la cultura no admite una definición meramente antropológica; precisa en cambio de un abordaje simbólico que permita dar cuenta del diverso y complejo universo de sentidos contenido en ella. La comunicación intercultural se da en la interacción, en una situación comunicativa concreta que obedece a las coordenadas de una configuración especio-temporal determinada.

Por ello, más que abordar la cultura desde una perspectiva estrictamente antropológica y/o sociológica, este artículo propone un concepto de cultura que se fundamente más bien en la sociología fenomenológica, privilegiando el proceso, es decir, la interacción misma, y donde las explicaciones que las condiciones históricosociales puedan ofrecer funcionen como una especie de marco goffmaniano que condicionen, mas no determinen, la dirección de la interacción.

En un intento por esclarecer lo que hemos anunciado anteriormente, nos referiremos a tres definiciones sobre la cultura que sirven de base para los objetivos que este artículo pretende. Como primera propuesta, retomamos la definición dada por Jorge González (1987) que concibe a la cultura como el principio organizador de la experiencia humana, es decir, el lugar en el que los individuos y grupos se posicionan, se reconocen, se definen y se relacionan. Por su parte, los Estudios Culturales permiten entender la cultura como un terreno efectivo donde se construye la hegemonía, y en el cual diversas corrientes ideológicas recrean distintos puntos de articulación, es decir, la cultura se instaura en las formas en las que las relaciones históricas de dominación inciden de forma articulada en la concepción de las identidades de los sujetos y grupos sociales involucrados. Por último, el concepto de cultura que propone Comaroff (1992) permite entenderla como la relación dialéctica entre estructura y práctica en la que se reproduce y transforma el carácter del orden social mismo.

Los tres enfoques anteriores nos acercan a las dimensiones cognitiva y comunicativa de la cultura, y es en la intersección de ambas dimensiones donde situamos a la interculturalidad. No obstante, creemos pertinente apropiarnos también de otras concepciones diversas de cultura como las propuestas por Freud (1927; 1974), Geertz (1987), González (1987), María Jesús Buxó i Rey (1990), García Castaño (1992), Bodlye (1994), Donald y Rattansi (1992), Triandis (1977) y Brislin 
(1981), con el objetivo de ofrecer un panorama más completo del concepto que nos ocupa.

La idea de cultura que presentamos intenta dar cuenta de los mecanismos de estructuración del mundo, de los modelos que sirven para dar sentido a la realidad. Por ello no podemos soslayar el sentido constructivo de la misma, su carácter cognitivo -mental- y comunicativo - relacional-, su estatuto pragmático y dialéctico, y la dimensión de saber común que permite justamente el mínimo de interacción necesaria para poner en marcha la noción de territorio simbólico, que es el lugar no sólo de las construcciones de los sentidos intersubjetivos, sino el ámbito de apropiación, interpretación y reinterpretación de los mismos.

Bodlye (1994) concibe a la cultura como algo que se transmite de generación en generación a través del aprendizaje; adquirir una cultura, por lo tanto, no significa nacer con ella, sino aprender los significados compartidos presentes en la misma, lo que permite de alguna manera pertenecer a ella y poseer una identidad. En este sentido, la cultura va estrechamente unida con el proceso de socialización primaria, que tiene lugar por medio de la familia, la escuela, la religión y los medios masivos de difusión, entre otras instancias. De esta concepción nos interesa remarcar el carácter adquirido, aprendido y compartido de la cultura. De ahí que cuando se hable de identidad cultural no se haga referencia a algo fijo sin más, sino y sobre todas las cosas a lo que compartimos, sea estático o dinámico. Nuestra postura se aleja, por tanto, de las primeras concepciones de la identidad, que la entendían como algo estático, esencial, algo con lo que se nace y se muere, sin posibilidad de cambio alguno. Por ello, la idea de cultura de Bodlye no implica en ningún caso la anulación de las diferencias individuales, sino que apunta a lo que compartimos en tanto nos ayuda a comunicarnos, y genera relaciones de pertenencia más allá de la identidad biológica o histórica con la que contemos. Lo interesante de esta definición es que incorpora el elemento comunicativo: la cultura necesita de la comunicación no sólo para transmitirse de generación a generación, sino que la propia existencia de la cultura, objetivada en prácticas sociales e interacciones, e incorporada por los sujetos, está mediada por procesos de comunicación.

Por su parte, Triandis (1977) concibe a la cultura como estrechamente relacionada con la identidad cultural. A esta última la entiende como el marco de 
referencia común que sirve de base para la comprensión del mundo y su funcionamiento, en tanto permite a partir de él interactuar con otras personas y elaborar expectativas y acontecimientos. Este marco de referencia se construye, por una parte, a través de las dinámicas sociales y culturales que se articulan con el poder y la ideología, pero por otra, se da y forma parte de las prácticas de uso y las comunidades interpretativas, cuyos significados se pactan.

Esto nos lleva a prestar atención a la definición que da Brislin (1981) sobre la cultura subjetiva, que es semejante a la de identidad cultural propuesta por Triandis. La cultura subjetiva se define como la respuesta de los sujetos a la parte del medio ambiente hecha por el hombre, o como la forma característica de un grupo de percibir su medio ambiente social.

La definición anterior tiene muchos puntos de contacto con el concepto de habitus de Bourdieu (1990), comprendido como el conjunto de disposiciones incorporadas que conducen a los sujetos a pensar, actuar y percibir de una forma en cierta medida determinada. Si entendemos el habitus como una forma interiorizada que "dispone" al individuo a actuar de una u otra manera (Bourdieu, 1990) en función de una serie de incorporaciones simbólicas a lo largo de su experiencia de vida, el concepto de respuesta manejado por Triandis encaja conceptual y metodológicamente con el de habitus para formar ambos una visión más completa de lo que el propio Triandis define como cultura subjetiva.

A pesar de que la cultura proporcione un marco de referencia desde donde los sujetos pueden comprender el mundo y comprenderse como individuos y sujeto sociales pertenecientes a un grupo, la respuesta de los individuos y grupos al medio ambiente construido por el hombre (que no son más que las propias relaciones que se producen entre ellos y las formas de comportamiento y acción que se desprenden de ella), está condicionada por ciertas mediaciones de los sentidos sociales, por las "disposiciones" de los individuos y los grupos a una acción determinada (Bourdieu, 1990).

Si partimos de que los sentidos sociales se hallan constituidos en núcleos de mediación o interpretación diferenciales en cuanto a grupos, pero en menor medida en cuanto a individuos, debemos de adaptar el concepto de cultura subjetiva al de 
cultura intersubjetiva ${ }^{3}$, y dar cuenta así de aquellas acciones o conocimientos que funcionan como respuestas al contacto medioambiental, pero que al mismo tiempo no pueden estar desvinculadas del todo de las condiciones estructurales -clivajes- de la sociedad donde tienen lugar.

La antropóloga María Jesús Buxó i Rey (1990) concibe a la identidad cultural como una construcción mental, es decir, como una estructura de orden simbólico que, según los postulados del psicoanálisis de Freud (1974), contiene al mismo tiempo el saber y el poder adquirido por los hombres y las organizaciones necesarias para "fijar" las relaciones entre ellos. Y las "organizaciones", en el contexto psicoanalítico, constituyen las articulaciones mentales que oscilan entre el ego -lo individual- y el superego -lo colectivo-, por ello la relación individuo-sociedad juega un papel importante en la construcción de dichas articulaciones.

En un sentido similar, la definición de cultura como proceso dada por Geertz (1987) permite indagar en las articulaciones mentales de las que hablaba Freud (1974). Geertz concibe a la cultura como una red de significaciones y sentidos que sirve para significar la vida, y esta idea encaja con la noción de que la cultura posee dos dimensiones dialécticas: la dimensión de la tradición, de lo que ya está dado y nos identifica, y la dimensión de la innovación, de lo que se construye en el quehacer cotidiano. Estas dimensiones empatan con la idea de la cultura como relación dialéctica entre estructura y práctica dada por Comaroff (1992).

Todo lo anterior nos hace proponer una concepción de cultura que retoma lo que García Castaño (1992) entiende por ésta: "totalidad de creencias aprendidas, herramientas y tradiciones compartidas por un grupo con la finalidad de dar continuidad, orden y significado a sus vidas". De esta manera, la cultura consta de las experiencias y productos acumulados por un grupo, y de ese hacer que las transforma del que habla Comaroff (1992).

\footnotetext{
3 El concepto de intersubjetividad puede ser abordado a partir de la propuesta sociofenomenológica de Alfred Schütz $(1971 ; 1979 ; 1993)$. El autor no se centra ni en el sistema social ni en las relaciones funcionales que se dan en la vida en sociedad, sino en la interpretación de los significados del mundo (lebenswelt) y las acciones e interacciones de los sujetos sociales. Del mundo conocido y de las experiencias intersubjetivas compartidas por los sujetos, se obtienen las señales, las indicaciones para interpretar la diversidad de símbolos.
} 
La cultura así entendida viene a ser en términos básicos lo que plantean Donald y Rattansi (1992): la forma en que las creencias, los rituales y las tradiciones son producidas a través de sistemas de significados, estructuras de poder e instituciones en que se repliegan. A esta definición podemos añadir que no sólo dichas tradiciones son "producidas", sino también re-producidas, re-creadas, entendidas, asumidas, interpretadas, re-interpretadas y re-significadas a través de los sistemas de significados que el poder articula, pero también mediante aquellos significados que entran a jugar en la arena pública de una manera distinta porque se construyen precisamente desde una zona fronteriza que mezcla e indefine justamente a la cultura como expresión identitaria, para asumirla como una construcción representativa, diferenciada y articulatoria.

Las siguientes líneas recuperan todo lo anterior con un fin particular: establecer algunas relaciones conceptuales entre la cultura y la comunicación intercultural, a partir del concepto de frontera, que entendemos puede dar cuenta de las disposiciones subjetivas y las condiciones estructurales que permean, ya sea para facilitar u obstaculizar, toda relación de interacción.

\section{La interculturalidad como proceso comunicativo}

La interculturalidad es, antes que cualquier otra cosa, una postura. Una postura híbrida, una tarea por hacer, una tarea inconclusa que plantea la necesidad de buscar caminos para la integración, la armonía y el desarrollo humano. Por ello, el ser intercultural se corresponde fundamentalmente no con la ejecución concreta y particular de estrategias o acciones encaminadas a tal fin, sino con el acto mismo de pensar y actuar conforme a un pensamiento intercultural.

La postura o actitud intercultural obedece a un sinfín de factores que, heredados, adquiridos $\mathrm{y} / \mathrm{o}$ construidos en un porcentaje indeterminado $\mathrm{y}$ dependiente en su mayoría de cada sujeto particular, afianzan la idea de la interculturalidad como un pendiente de las relaciones humanas y las políticas de gestión social.

El hacer y el saber intercultural nos coloca en el punto medio de los ámbitos de tensión entre lo ideal y lo real, entre el fin perseguido y la realidad que lo produce y lo acoge. Su falta de concreción no comporta necesariamente inoperancia, si no más 
bien la clara convicción de que se trata de un camino en constante construcción, cuyo recorrido es en la misma medida fruto de un pensar y un hacer intercultural.

Como proceso, la interculturalidad pasa de manera indefectible por la comunicación o para ser más exactos, es comunicación intercultural. La comunicación, comprendida como interacción, es vínculo entre sujetos, es relación antes que cualquier otra cosa. Y por ello, la interculturalidad no puede ser otra cosa que comunicación intercultural, y apuesta tanto a la competencia como a la cooperación y la disposición que permiten a los sujetos compartir saberes y acciones, poner en común o en contacto la urdimbre de significados que dan sentido a la vida cotidiana, a sus prácticas y a sus representaciones simbólicas.

En la medida en que esta comunidad de vida sea mayormente compartida, la posibilidad de incrementar la eficacia de la comunicación y en particular de la comunicación intercultural será también mayor, y en consecuencia mayor posibilidad habrá que emisor y receptor entiendan, asuman y aprehendan recíprocamente el sentido que tienen las cosas para cada uno de ellos.

En el proceso de comunicación intercultural, dos instancias "culturales”, dos universos simbólicos e interpretativos diferentes entran en contacto e intercambian, no sin conflictos, informaciones. La semiosis es el mecanismo por excelencia que permite la entrada, la salida y el entrecruzamiento de la información necesaria para la comunicación.

Como ya hemos advertido, no entendemos a la comunicación intercultural como una comunicación armónica ni simétrica; se trata, justamente por las diferencias de rol y posición que en un espacio físico o simbólico comportan los sujetos implicados, así como sus comunidades de sentido, de una comunicación mayormente conflictiva que debe tender, en su ideal, al mantenimiento y consolidación de su eficacia, pero no a la anulación de sus conflictos ${ }^{4}$.

\footnotetext{
4 La eliminación de los conflictos comunicativos implicaría por una parte la ausencia de dinamismo en la interacción, lo que equivaldría a decir ausencia de intercambio y "nutrición" de los sistemas semióticos en contacto, por un lado, y eliminación de las diferencias necesarias para echar a andar la dinámica interaccional, por el otro.
} 
Estamos conscientes que al definir a la interculturalidad como postura, es decir, como una postura subjetiva, humana, estamos hablando de una especie de "disposición", de un "disponerse a" consciente y autorreflexivo que implica necesariamente un conocimiento vasto del sí mismo, pero en ningún momento la posesión y ejercitación de este conocimiento impide la presencia de conflictos y contradicciones incluso al interior de un mismo sujeto.

Lo diferente es, en ese sentido, la contraparte natural del desarrollo de la postura intercultural; la diferencia permite pensar la otredad y la alteridad, y permite actuar consecuentemente a partir de ellas. Se trata de enriquecer el acto comunicativo mediante el intercambio y el reajuste de los datos de entrada y de salida; anular la diferencia sería en todo caso dar muerte al sistema y a la interacción.

Pero gracias a la misma dinámica de la interacción, la anulación no puede darse de manera total, aun cuando haya empeño en la tarea. El concepto de "frontera interna" que desarrollaremos a continuación posee una instancia constitutiva en la que los "reajustes" en función de la estabilización en una interacción conflictiva no pueden darse del todo.

\section{Comunicación intercultural y fronteras internas}

En párrafos anteriores hemos hecho referencia a que la pretendida reciprocidad entre los sujetos que participan de una situación de comunicación intercultural plantea un problema irresoluble hasta el momento. Se trata de poner sobre la mesa el problema del poder y de las hegemonías, el de las contaminaciones y/o resistencias, el de las diferencias culturales, y el de frontera. La frontera es una zona difusa de constantes interpenetraciones, una instancia real o simbólica que a veces articula y otra veces separa, pero que casi siempre queda confinada a un perímetro que divide, segmenta, distingue y separa identidades, grupos, representaciones, significados y, al fin y al cabo, culturas.

Lo anterior nos lleva a considerar las relaciones entre frontera y ruptura, ambos conceptos tomados en su doble condición dialéctica de límite y cambio. Tanto la ruptura como la frontera permiten pensar a la comunicación intercultural como un proceso no exento de tensiones, esto es, como un proceso conflictivo en el que se impone la necesidad -como ya hemos anunciado- de ajuste, de acomodación, de 
negociación de los significados implicados para poder vencer los obstáculos de la ineficiencia comunicativa.

El concepto de frontera se propone como punto de partida para indagar tanto en los ámbitos como en los elementos que pueden constituir el núcleo de conflictividad y/o integración en la comunicación intercultural. Para ello, tomamos en cuenta dos categorías conceptuales de la frontera: la primera, aquella que define a la frontera como límite o demarcación que obstaculiza la comunicación en tanto constituye la zona de resistencia donde lo irrenunciable se defiende; y la segunda, que entiende a la frontera como zona de ruptura, rendición y negociación de las identidades sociales y culturales, es decir, como espacio físico y mental contaminado, híbrido, permeable y “dispuesto” a la integración.

La frontera, entonces, cuando se manifiesta en el ámbito de la identidad, puede dar paso a otro concepto: el de "identidades fronterizas", mismo que sólo definiremos en este artículo en aras de ampliar la comprensión de nuestro concepto de frontera interna.

Es necesario partir de que el concepto de frontera interna está asociado a la subjetividad de los individuos y los grupos. Por ello cuando hablamos de identidades fronterizas nos referimos a aquellos elementos o espacios que dentro de una identidad social y cultural acotada permiten dar cuenta de dos cosas: por una parte, de los "límites" que desde la propia identidad obstaculizan o impiden "compartir" los significados de vida de los sujetos, debido justamente a que constituyen el grado de identidad irrenunciable que rescata, defiende y/o compite por la diferenciación como manifestación de la existencia real y sustancial de la distinción y la pertenencia; y por la otra, del lugar común de las transiciones, las rendiciones y las articulaciones que facilitan la negociación de los valores y significados de vida.

Este grado de irrenunciabilidad es precisamente lo que impide el reajuste total en una interacción, e impide por tanto la anulación real de los elementos de conflicto en una situación de comunicación dada. Lo irrenunciable no tiene razón de ser, simplemente ES; no se cuestiona ni se reflexiona sobre ello porque no se asienta sobre bases lógicas, sino ónticas y metafísicas. 
Lo irrenunciable pertenece al campo de "lo correcto" y eso es lo que impide su negociación. El sentido de lo correcto varía de sujeto a sujeto, y también de cultura a cultura; en función de él se construyen y legitiman los principios de la moral, los sentidos de pertenencia, los modos o formas de vida, lo aceptable y lo inaceptable, lo posible y lo imposible.

Ya sea en términos de biografía y experiencia individual -lo que Schütz (1993) denominaría "situación biográfica"- o en términos de identidad social, cultural y colectiva, el grado de irrenunciabilidad está presente en toda interacción y determina la "disposición" de los sujetos al reajuste o reacomodo necesario (el lugar de transiciones y rendiciones) para el mantenimiento de la comunicación.

La disposición debe entenderse en términos de "habitus" como una especie de actitud que es "incorporada" a partir de las pautas de acción real que son marcadas desde el ámbito estructural, pero al mismo tiempo desde la posibilidad y habilidad del sujeto para transformar dichas disposiciones y de "incorporarlas" de otras maneras a sus ámbitos de acción y significados de vida. El primer tipo de disposiciones es privativo del campo social; el segundo tiene su punto de partida tanto en el campo de lo social como en el campo de la interacción particular y concreta donde tiene lugar el encuentro comunicativo.

Teniendo en cuenta que en una interacción no sólo se ponen en juego los elementos que estructuran real y simbólicamente la identidad y la posición social de los sujetos, sino que también se representan éstos mediante una fachada o marco (Goffman, 1972), así como a través de los roles que cada uno de los sujetos está llamado a representar en ella, podemos pronosticar que lo irrenunciable puede sufrir alteraciones durante el curso de la interacción, e incluso -diríamos- puede ser disminuido y/o reducido en aras de estabilizar la interacción, de hacerla menos conflictiva; sin embargo, consideramos que justamente por su cualidad óntica, por su existencia per se, lo irrenunciable sólo puede ser eliminado o reconvertido si existe un proceso de reflexión suficientemente consciente como para poder cuestionarlo y transformarlo, dicho sea de paso en otra irrenunciabilidad.

En resumen, el concepto de frontera interna, articulado tanto desde los ámbitos de irrenunciabilidad como desde los ámbitos de ruptura y negociación, 
permite abordar la conflictividad inherente a la comunicación intercultural, misma que aparece íntimamente vinculada a la diferencia y a la diversidad como elementos constitutivos de una cultura.

Por ello, la relación entre cultura y comunicación intercultural puede abordarse, precisamente, a partir de este concepto de frontera. Puesto que toda situación de comunicación intercultural se constituye como un proceso inconcluso, nos parece pertinente entender el concepto de frontera más como el conjunto de límites simbólicos contenidos en las representaciones de los sujetos y grupos sociales, que como el conjunto de barreras físicas que separan y diferencian a estos sujetos y grupos.

\subsection{Algunas concepciones de la frontera}

La frontera se concibe como un límite mental o imaginario más que real o físico, debido al ordenamiento y/o reordenamiento al que somete a las dimensiones de la vida: el tiempo y el espacio, los comportamientos y las acciones, los deseos, las aspiraciones y las frustraciones.

Gran parte de los estudios acerca de la frontera se insertan en disciplinas como la economía, la demografía, la geografía y la política. Sin negar la importancia y pertinencia de este tipo de estudios, consideramos que carecemos de trabajos que pongan énfasis en el mundo de lo subjetivo, el mundo de las representaciones, los universos simbólicos y los sentidos de la vida. Sin conocimiento acerca de este otro mundo, menos tangible y más difícil de objetivar y de apreciar a primera vista, no podremos comprender cómo es que se construyen las relaciones de negociación y/o disputa entre los mundos del "nosotros" y de los "otros".

Asimismo, consideramos a las fronteras como movibles y permeables, lo cual nos acerca al concepto de identidades fronterizas, que como ya hemos dicho anteriormente, entendemos puede dar cuenta de procesos constantemente producidos y re-producidos, significados y re-significados, como productos de las relaciones con los "otros".

Por otra parte, concebimos a la frontera en su doble condición de frente cultural (González, 1987) y territorio del habitus (Bourdieu, 1980; 1990; 1999). De 
esta doble articulación surge lo que hemos denominado frontera interna. El concepto de frentes culturales sirve como herramienta metodológica y teórica para pensar y a explicar empíricamente los modos históricos, estructurales y cotidianos en los que se construye una urdimbre de relaciones de hegemonía en una sociedad determinada. Por su parte, el habitus permite dar cuenta de las formas de interiorización o incorporación de disposiciones para la acción.

La tercera concepción de frontera que retomamos viene dada por la dimensión de lucha y sentido entre dos tiempos. Aquí los conceptos de identidad, el "nosotros" y el "ellos", pueden actualizarse con el concepto de lucha o frente cultural tanto desde el freno del habitus o los clivajes, como desde el concepto de praxis social, entendido como dinámica, proceso, quehacer cotidiano, articulación de un tiempo/lugar y otro. Por ello, las fronteras, como espacios sociales altamente complejos y cruzados por múltiples vectores, nos ofrecen un buen pretexto para poder entender cómo se construye el sentido de lo propio y lo ajeno; del "nosotros" y de los "otros".

\subsection{Factores estructurales, identidades fronterizas e interculturalidad}

Los factores estructurales a los que hemos hecho referencia no son otros que los que Briones y Siffredi (1989) denominan clivajes; dichas investigadoras consideran a la clase, la raza, la etnia, la religión, el sexo, la edad y demás factores estructurales y estructurantes de la identidad como construcciones sociales que se articulan a partir de una dinámica de agregación y desagregación. Estas construcciones son concebidas por las autoras como condiciones de materialidad, es decir, objetivas, tangibles, que pueden palparse nítidamente como elementos ejes de las identidades, conformando a partir de ellas el universo de sentido donde se posicionan los territorios de yo, del nosotros y de lo ajeno.

Estos mismos clivajes o anclajes son los factores que constituyen la experiencia de vida del sujeto y desde los cuales éste proyecta o irradia su ser para sí mismo, pero sobre todo para dar sentido y valor a la relación existente para con los otros, sus otros, y el mundo que le rodea. 
La noción de acción intercultural enfocada en la utopía, pero no en la imposibilidad de la construcción del trayecto hacia ella, implica remitirnos a su carácter pragmático, a su presupuesto interactivo, dialógico y necesariamente dialéctico. Esto es lo que le da su estatuto de acción. De ahí que una acción intercultural esté siempre encaminada hacia una serie de posturas y/o comportamientos donde los "hablantes" estén presentes en y con su diferencia, pero sobre todo en y con la posibilidad y habilidad para "compartir".

Al respecto, señala Lotman (1994: 118): “el impulso para la interacción no resulta del parecido o del acercamiento (estadial, de sujeto y motivos, genéricos, etc.), sino de la diferencia”. Es decir, la diferencia como motor de la interacción en tanto búsqueda de lo ajeno y como herramienta fundamental del desarrollo cultural (Zhirmunski, citado en Lotman, 1994: 118), es lo que nos lleva a considerar a la acción intercultural como una acción de competencia comunicativa.

A partir de lo anterior, trabajar con la categoría de límite o irrenunciabilidad presente en las identidades fronterizas, es decir, con aquello que definitiva y significativamente plantea la tensión entre lo que somos y pensamos que somos diferencialmente, y lo que impide desde esta distinción la interrelación con el otro, resulta de vital importancia para comprender las relaciones entre cultura y comunicación intercultural.

\section{Interculturalidad y competencias comunicativas}

Como proceso interactivo, la comunicación permite llevar a cabo la interculturalidad, la hace manifiesta, objetivable; y como principio de contacto, la comunicación contribuye a la interculturalidad en tanto que puede privilegiar -en contextos de negociación o conflicto- el respeto entre sujetos.

Comprender las relaciones interculturales en una situación comunicativa concreta supone comprender la cultura de los dos mundos en contacto. Si definimos a la cultura como la malla de significados o sentidos que dan sentido a la vida misma (Weber) en la forma de "programas" computacionales (Geertz) que en la práctica se convierten en sistemas de valores y normas que rigen la acción (Giddens), la comunicación intercultural se realiza donde hay contacto entre dos o más de esos 
entramados de significados y sentidos, y cuando un grupo comienza a entender, en el sentido de asumir, el significado y el valor de las cosas y objetos para los "otros".

Esto no quiere decir, que en la comunicación intercultural se asimilen y acepten de manera conclusiva los significados y sentidos de vida del otro o de lo otro, sino que la comprensión efectiva de dichos significados propicie o facilite el entendimiento necesario para disminuir el grosor de las diferencias o los límites de esa irrenunciabilidad que hemos descrito. Se trata, más que nada, de accionar las condiciones para que, a través del entendimiento y la explicación de los sentidos del otro se genere una zona porosa, común, permeable, donde las diferencias enriquezcan la interacción humana.

Parafraseando a Casmir (1993), la construcción de una tercera cultura a partir de dos culturas iniciales no puede ser la suma de ellas mismas, sino la creación de un tercero otro, de una nueva diferencia donde se estructuren y objetiven intercambios convergentes. La tercera cultura no es más que el perímetro intercultural donde las posturas interculturales como el respeto, la tolerancia, la curiosidad positiva, la voluntad y el deseo de cooperación armónica puedan promover, más allá de las diferencias constitutivas, el intercambio y la comunicación.

Este encuentro comunicativo eficaz cambiaría necesariamente el rostro de la eficacia como concepto. La eficacia, entonces, lejos de circunscribirse al hecho de reconocer y compartir los códigos de la comunicación, se enfrentaría al reto de “compartir", reconocer y respetar los diferentes sentidos de las diferentes matrices codiciales que intervienen. Sólo así, la comunicación se tornará eficaz. Es decir, la comunicación es eficaz si se logra un grado de comprensión aceptable para los interlocutores, o lo que es lo mismo: si éstos logran compartir suficientemente las significaciones de lo que dicen.

La búsqueda de la eficacia intercultural, por tanto, conduce a crear competencia comunicativa y ésta a establecer pautas asertivas que orienten la experiencia comunicativa hacia una experiencia "compartida". La competencia comunicativa intercultural no debe ser concebida tanto como un conjunto de saberes y conocimientos, sino más bien como un conjunto de disposiciones hacia la tolerancia, respeto, convivencia y comprensión de lo otro, de lo ajeno. 
Teniendo en cuenta que los participantes en un encuentro intercultural interactúan apoyándose en suposiciones culturales propias, mismas que actúan como pantallas perceptuales de los mensajes que intercambian, es fácil suponer que el marco de referencia cultural en el que cada comunicador interpreta los mensajes puede variar de una mínima hasta una máxima diferencia (en ocasiones, algunas de ellas suelen ser obvias, mientras que otras pueden ser más sutiles); por ello, el éxito o fracaso de la interacción dependerá, en gran medida, de la familiaridad de los participantes con los antecedentes de su interlocutor, las percepciones de las diferencias que los separan y la reciprocidad del propósito. Sólo así, la comunicación pasará de ser un acto individual a ser una experiencia "compartida".

Es en este sentido que consideramos que en esta experiencia compartida, no necesariamente -insistimos- aceptada y asimilada, se dan los primeros pasos para el entendimiento y la comprensión de y hacia lo diferente.

\section{Cierre reflexivo}

Como se ha podido observar, el establecimiento de puentes y negociaciones de sentido en una situación de comunicación intercultural encuentra como límite, limitante pero obligado, a la cultura, es decir, a las representaciones particulares que despliegan los sujetos en dicha situación de interacción, a los lugares desde donde éstos se piensan, actúan y se comunican. Estos límites dan como resultado el trazo de una línea fronteriza que más que física aparece como una barrera mental desde la que se definen y perciben los sujetos, y al mismo tiempo desde donde definen y perciben a los demás. "Lo propio es siempre una condición de lo ajeno", dice Bilbeny (2005), de ahí que debamos preguntarnos de qué modo los sujetos pueden vivir lo propio para que lo ajeno también tenga lugar y se posibilite el diálogo y la negociación entre ellos.

Partir de un concepto esencialista y estático de cultura dificulta la reflexión en torno a las posibilidades de una comunicación intercultural eficaz. Si bien la cultura se traza como un límite, no debemos considerar como imposible el establecimiento de puentes de comunicación, de espacios porosos (fronteras internas, como las hemos llamado) donde se facilite o propicie el intercambio y la negociación de sentidos sobre el mundo, sobre el "nosotros" y los "otros". 
Cultura y comunicación intercultural son conceptos estrechamente ligados. Más bien se necesitan mutuamente. No puede existir cultura sin comunicación entre sujetos, pues la interacción es la base de toda sociedad; y por otra parte, no puede existir comunicación -y concretamente comunicación intercultural- sin tomar en cuenta que los sujetos que se comunican son portadores de una cultura específica, un “desde dónde", unos "lentes" desde los cuales miran y comprenden el mundo, desde los cuales actúan, se comportan, se piensan a sí mismos y a los demás.

En este caso, hemos articulado la relación entre cultura y comunicación intercultural a partir del concepto de frontera interna, que permite pensar esas zonas porosas, permeables, que median toda interacción entre sujetos, y en las cuales es posible observar los límites y obstáculos pero también los elementos facilitadores o propiciadores de la interacción entre sujetos distintos.

\section{Bibliografía:}

BILBENY, Norbert (2005) Uno mismo y los otros. De las experiencias existenciales a la interculturalidad. Barcelona: Herder.

BOURDIEU, Pierre (1980) El sentido práctico. Madrid: Taurus.

_ (1990) Sociología y cultura. México: Fondo de Cultura Económica.

_ (1999) Las reglas del arte. Génesis y estructura del campo literario. Madrid: Anagrama.

BODLYE, John H. (1994) “An Anthropological Perspectives”, en revista Cultural Anthropology: Tribes, Status and the Global System. Artículo en línea, disponible en la página http://www.wsu.edu:8001/vcwsu/commons/topics/culture/culturedefinitions/bodley-text.html (Fecha de consulta: octubre 2005).

BRIONES, Claudia y SIFFREDI DE LA NATA, Alejandra (1989) "Discusión introductoria sobre los límites teóricos de lo étnico" en Cuadernos de Antropología, Núm. 3. Buenos Aires: UNL-Eudeba, 5-24.

BRISLIN, Richard W. (1981) Cross-Cultural Encounters: Face-to-Face Interaction, New York: Pergamon Press.

BUXÓ Y REY, María Jesús. (1990) "Vitrinas, cristales y espejos: Dos modelos de identidad en la cultura urbana de las mujeres Quiche de Quetzaltenango”. En 
José Alcina Franch (compilador), Indianismo e indigenismo en América. Madrid: Alianza, pp. 134 .

CASMIR, F. L. (1993) "Third-Culture Building: A Paradigm Shift for International and Intercultural Communication", en DEETZ, S. A. Communication Yearbook, 16, Londres: Sage, pp. 407-428.

COMAROFF, John (1985) Body of Power, Spirit of Resistence, Chicago: University of Chicago Press.

COMAROFF, John y COMAROFF, Jean (1992) "Of totemism and Etnicity". En Ethnography and the Historical Imagination, Wetview Press, Boulder.

DONALD, J. y RATTANSI, A. (1992) Raza, cultura y diferencia. Londres: Sage.

FREUD, Sigmund [1924] (1974) El porvenir de la ilusión. En Psicología de masas. Madrid: Alianza, 4ta edición.

GARCÍA CASTAÑO, F. J. y PULIDO Moyano, R. A. (1992) "Educación Multicultural y Antropología de la Educación”, en FERMOSO, P. (ed.) Educación Intercultural: la Europa sin fronteras. Barcelona: Narcea, pp. 35-69.

GEERTZ, Clifford (1987) La interpretación de la cultura. México: Gedisa

GOFFMAN, Erving (1972) La presentación de la persona en la vida cotidiana, Buenos Aires: Amorrortu Editores.

GONZÁLEZ, Jorge A. (1987) “Los frentes culturales: culturas, mapas, poderes y luchas por las definiciones legítimas de los sentidos sociales de la vida” en Estudios sobre las culturas contemporáneas, Época I, Núm. 3. Colima: Universidad de Colima, 5-44.

LOTMAN, Iuri (1994) "Para la construcción de una teoría de la interacción de las culturas (el aspecto semiótico)”. En Criterios No. 32, Cuarta Época, juliodiciembre, pp. 117-130.

_ (1996) La Semiosfera I. Semiótica de la cultura y del texto. Madrid: Cátedra.

_ (1998) La Semiosfera II. Semiótica de la cultura y del texto. Madrid: Cátedra. _ (2000) La Semiosfera III. Semiótica de la cultura y del texto. Madrid: Cátedra.

SCHÜTZ, Alfred (1971) Fenomenología del mundo social. Introducción a la sociología comprensiva, Buenos Aires: Paidós.

_ (1979) El problema de la realidad social, Buenos Aires: Paidós

_ (1993) La construcción significativa del mundo social, Barcelona: Paidós

TRIANDIS, Harry (1977) "Subjective Culture and Interpersonal Relations Across Cultures," in L. Loeb-Adler (ed.), Issues in Cross-Cultural Research, Annals of the New York Academy of Sciences, 285, pp. 418-434.

WATZLAWICK, Paul (et.al.) (1971) Teoría de la comunicación humana. Interacciones, patologías y paradojas, Buenos Aires: Tiempo Contemporáneo. 